\title{
TGM2 knockdown reverses cisplatin chemoresistance in osteosarcoma
}

\author{
CUIYUN LI ${ }^{1}$, JING CAI $^{2}$, FUGUI GE ${ }^{3}$ and GUILONG WANG ${ }^{4}$ \\ Departments of ${ }^{1}$ Pathology and ${ }^{2}$ Neurosurgery, Linyi People's Hospital; ${ }^{3}$ Department of Surgery, Linyi Women's and \\ Children's Hospital; ${ }^{4}$ Department of Orthopedics, Linyi People's Hospital, Linyi, Shandong 276000, P.R. China
}

Received November 22, 2017; Accepted April 18, 2018

DOI: $10.3892 /$ ijmm.2018.3753

\begin{abstract}
In the past decades, chemotherapy has resulted in improved outcomes for patients with osteosarcoma. However, resistance to chemotherapy often leads to poor prognoses. Cisplatin is a standard drug for osteosarcoma therapy, and chemoresistance to cisplatin in osteosarcoma limits the effectiveness of chemotherapy drugs. Transglutaminase 2 (TGM2) is a member of the transglutaminase family, and it is reported to be associated with chemoresistance in various types of cancer. The present study aimed to investigate the function of TGM2 in regulating chemosensitivity of osteosarcoma cells to cisplatin. For in vitro experiments, a cisplatin-resistant osteosarcoma cell line (Saos2-CIS-R) was established, and TGM2 was demonstrated to be upregulated in the resistant Saos2-CIS-R cells compared with the normal Saos 2 cells. The present study also revealed that TGM2 was associated with chemoresistance to cisplatin in osteosarcoma cells, and knockdown of TGM2 enhanced their chemosensitivity. In addition, TGM 2 was demonstrated to affect the chemosensitivity of osteosarcoma cells via regulation of the activation of mitogen-activated protein kinase and AKT serine/threonine kinase pathways. Expression of BCL2 apoptosis regulator, BCL2 associated X and caspase-3 was also involved in chemoresistance development in osteosarcoma. For in vivo experiments, a mouse model was used to detect that the cisplatin sensitivity of Saos2-CIS-R cells was reversed following TGM2 knockdown. Taken together, the present data suggested a potentially important role for TGM 2 in the regulation of osteosarcoma chemosensitivity. TGM2 might therefore serve as a therapeutic target for osteosarcoma.
\end{abstract}

Correspondence to: Dr Guilong Wang, Department of Orthopedics, Linyi People's Hospital, 27 Jiefang Road, Linyi, Shandong 276000, P.R. China

E-mail: cj7012299@163.com

Key words: transglutaminase 2, osteosarcoma, chemoresistance

\section{Introduction}

Osteosarcoma is the most common primary malignant bone tumor in children and young adults in the USA (1). In the past decades, several studies have reported improved outcomes for patients with osteosarcoma with the addition of neoadjuvant and adjuvant chemotherapy, and long-term survival for localized patients now approaches $70 \%(2,3)$. Despite the great progress in treating osteosarcoma, resistance to chemotherapy often results in metastasis and recurrence in patients with osteosarcoma, which leads to poor prognoses (4). Cisplatin is a standard osteosarcoma therapeutic agent that interacts with nucleophilic $\mathrm{N}^{7}$ sites of purine bases in DNA to induce DNA damage and subsequent cell death (5). However, the development of chemoresistance to cisplatin in osteosarcoma limits the effectiveness of chemotherapy drugs and the long-term survival rate for osteosarcoma patients with metastatic or recurrent disease remains poor (6).

Chemoresistance is acquired by tumors during chemotherapy treatment, even in tumors which were initially sensitive to the drug. This adaptive response could be due to regulation of expression of one or several genes and activation of related signaling pathways (7). Novel agents that target specific molecular alterations in tumors have been developed during recent decades, and these drugs have demonstrated encouraging results in restoring chemosensitivity (7).

Transglutaminase 2 (TGM2) is a member of the transglutaminase family. TGM2 is a multifunctional protein which is involved in cell apoptosis and extracellular matrix degradation $(8,9)$. Aberrant expression of TGM2 has been observed in multiple types of cancer cells and is associated with poor drug response and poor patient survival $(10,11)$. In vitro and in vivo experiments have demonstrated that TGM2 may be a potential therapeutic target for chemoresistant ovarian cancer (12). A recent study suggested a new antiapoptotic function of TGM2, with TGM2 inhibiting osteosarcoma cell apoptosis via regulating expression of BCL2 associated X (Bax) and the release of cytochrome $\mathrm{C}$ under hypoxia conditions (13). However, the role of TGM2 may differ in different tumor cell lines, and whether it is associated with the chemoresistance of osteosarcoma cells remains unknown $(14,15)$.

To better understand the effect of TGM 2 on chemoresistance, and provide a potential novel method of osteosarcoma treatment, in vitro and in vivo models were used to investigate 
the role of TGM 2 on chemoresistance by regulating the AKT serine/threonine kinase (Akt) and mitogen-activated protein kinase (MAPK) pathways. Knockdown of TGM2 was also demonstrated to successfully reverse chemoresistance to cisplatin in osteosarcoma.

\section{Materials and methods}

Cell culture and establishment of cisplatin-resistant cells. The human osteosarcoma cell line Saos2 was purchased from the American Type Cell Culture (Manassas, VA, USA). Cells were cultured in DMEM medium (HyClone; GE Healthcare Life Sciences, Logan, UT, USA) supplemented with $10 \%$ fetal bovine serum (FBS; HyClone; GE Healthcare Life Sciences) at $37^{\circ} \mathrm{C}$ in a $95 \%$ humidified and $5 \% \mathrm{CO}_{2}$ incubator. Cells were incubated with different concentrations of cisplatin (Sigma-Aldrich; Merck KGaA, Darmstadt, Germany). The concentration was stepwisely increasing to establish cisplatin-resistant cells (from 0.1 to $1 \mu \mathrm{g} / \mathrm{ml}$ ). Cells were cultured under each concentration of cisplatin for 7 passages. After 1 year, cells growing in $1 \mu \mathrm{g} / \mathrm{ml}$ cisplatin were designated as cisplatin-resistant cells and termed Saos2-CIS-R.

TGM2 knockdown and overexpression. To downregulate the expression of TGM2, Saos2-CIS-R cells were transduced with lentiviral particles containing short hairpin RNA (shRNA) against TGM2 (sh-TGM2; purchased from GeneChem Co., Ltd., Shanghai, China). A green fluorescent protein-tagged lentiviral vector containing a scrambled shRNA was transduced as a negative control. For TGM2 overexpression, Saos2 cells were transduced with lentiviral particles encoding for the full-length TGM2 gene (TGM2; purchased from GeneChem Co., Ltd.). Vectors were propagated on HEK293T cells (American Type Culture Collection), purified and titered, then stored at $-80^{\circ} \mathrm{C}$ prior to use. All transduced cells were subsequently exposed to $3 \mu \mathrm{g} / \mathrm{ml}$ puromycin (Sigma-Aldrich; Merck KGaA, Darmstadt, Germany) for 4 weeks post-infection to remove non-transduced cells, and then maintained in $1 \mu \mathrm{g} / \mathrm{ml}$ puromycin. The expression of TGM2 in all cells was confirmed by western blotting.

Cell viability and cisplatin sensitivity assay. Cells were seeded on 96-well plates at a density of $5 \times 10^{3}$ cells per well. Cell Counting Kit-8 (CCK-8; Dojindo Molecular Technologies, Inc., Kumamoto, Japan) was used to measure cell viability following treating cells with different concentrations of cisplatin. After treatments, the culture medium was removed, and cells were washed with PBS. A total of $100 \mu \mathrm{l}$ fresh medium with $10 \mu \mathrm{l}$ of CCK-8 solution was added to each well for $2 \mathrm{~h}$ at $37^{\circ} \mathrm{C}$. Optical density (OD) was measured at $450 \mathrm{~nm}$ using a microplate reader (BioTek Instruments, Inc., Winooski, VT, USA). The OD readings of the treatment groups were divided by their corresponding control readings to obtain the ratio of cell viability.

Cell apoptosis assay. Cells were seeded in 6-well plates at a density of $1.5 \times 10^{5}$ cells per well. Then cells were treated with different concentrations of cisplatin for $24 \mathrm{~h}$, as indicated. Cells were harvested following cisplatin treatments, washed twice with cold PBS and centrifuged. The supernatants were discarded and the cells were resuspended in 1XAnnexin-binding buffer. A total of $5 \mu \mathrm{l}$ Annexin V-allophycocyanin (APC) or Annexin V-allophycocyanin (FITC) solution (BD Biosciences, Franklin Lakes, NJ, USA) was added to the cells at room temperature for $15 \mathrm{~min}$, then $5 \mu \mathrm{l}$ propidium iodide (PI) solution (BD Biosciences) was added. The ratio of apoptotic cells (\% Annexin V-APC or Annexin V-FITC positive cells per total) was measured by flow cytometry (BD FACSDiva version 8.0.1; BD Biosciences).

Reverse transcription-quantitative polymerase chain reaction $(R T-q P C R)$. Total RNA was isolated using TRIzol reagent (Thermo Fisher Scientific, Inc., Waltham, MA, USA) following the manufacturer's instructions. cDNA was generated by reverse transcription of $1 \mu \mathrm{g}$ total RNA using the 1st Strand cDNA Synthesis kit (Takara Biotechnology Co., Ltd., Dalian, China). Relative gene expression was determined by qPCR using the SYBR Premix Ex Taq kit (Takara Biotechnology Co., Ltd.). The cycling conditions were 40 cycles of $95^{\circ} \mathrm{C}$ for $5 \mathrm{sec}$ and $60^{\circ} \mathrm{C}$ for $34 \mathrm{sec}$. The ABI Prism 7500 Fast Real-Time PCR system (Applied Biosystems; Thermo Fisher Scientific, Inc.) was used to perform the PCR experiments and to analyze the results. Primers were designed and selected using BLAST (National Institutes of Health, Bethesda, MD, USA), and are listed in Table I. Relative fold changes in mRNA expression were calculated using the $2^{-\Delta \Delta \mathrm{Cq}}$ method (16).

Western blot analysis. Cells were washed twice with cold PBS, then total protein was extracted with RIPA lysis buffer (Beyotime Institute of Biotechnology, Shanghai, China). Protein concentration was quantified by a bicinchoninic acid protein assay kit (Beyotime Institute of Biotechnology, Shanghai, China). A total of $20 \mu \mathrm{g}$ protein (for each sample) was loaded and separated by $10 \%$ or $12.5 \%$ SDS-PAGE, then transferred to polyvinylidene fluoride membranes (EMD Millipore, Billerica, MA, USA). The membranes were blocked with $5 \%$ fat-free milk for $1 \mathrm{~h}$ at room temperature and incubated with primary antibodies overnight at $4^{\circ} \mathrm{C}$ (anti-TGM2, anti-Bcl-2, anti-Bax, anti-akt, anti-p-akt, anti-caspase-3. anti-cleave-caspase-3 and the MAPK family antibody kits; cat. nos. 3557S, 3498, 5023, 8202S, 9926T, 9910T and 4060S; 1:1,000 dilution; Cell Signaling Technology, Inc., Danvers, MA, USA). Following three washes with TBS/ $0.1 \%$ Tween-20 (TBST), the membranes were incubated with anti-rabbit or anti-mouse immunoglobulin $\mathrm{G}$ for $1 \mathrm{~h}$ at room temperature (cat. nos. 7076 and 7074; 1:5,000 dilution; Cell Signaling Technology, Inc.) and visualized using an enhanced chemiluminescence system (PerkinElmer, Inc., Waltham, MA, USA). Positive immunoreactive bands were densitometrically quantified (Quantity One 1-D, version 4.6.9; Bio-Rad Laboratories, Inc., Hercules, CA, USA) and normalized to GAPDH.

Animal experiments. All animal operations were approved by the Animal Ethics Committee of Linyi People's Hospital (Linyi, China; Approval no. 2016-A036). A total of 40 four-weeks-old female nude mice (BALB/c, nu/nu; SIPPR-BK Laboratory Animal Co. Ltd, Shanghai, China) were housed under pathogen-free conditions at $18 \sim 22^{\circ} \mathrm{C}$ and $50 \%$ humidity; The mice had free access to food and water and exposed to the natural light-dark cycle. Cells (Saos2-CIS-R, 
Table I. Sequences of primers used in the quantitative polymerase chain reaction analysis.

\begin{tabular}{lll}
\hline Gene & Primer & \multicolumn{1}{c}{ Sequence ('5-3') } \\
\hline Bcl-2 & Forward & GAACTGGGGGAGGATTGTGG \\
Bcl-2 & Reverse & CCGTACAGTTCCACAAAGGC \\
Bax & Forward & CCAGAGGCGGGGTTTCAT \\
Bax & Reverse & GGAAAAAGACCTCTCGGGGG \\
GAPDH & Forward & ACCACCATGGAGAAGGCTGG \\
GAPDH & Reverse & CTCAGTGTAGCCCAGGATGC
\end{tabular}

Bcl-2, BCL2 apoptosis regulator; Bax, BCL2 associated X.

scramble and sh-TGM2) were cultured, counted and resuspended in PBS at a final concentration of $2 \times 10^{8}$ cells $/ \mathrm{ml}$. Mice were anesthetized with $1.5 \%$ pentobarbital sodium $(40 \mathrm{mg} / \mathrm{kg})$ and then injected with $50 \mu \mathrm{l}$ of cell suspension into the proximal tibia. When the tumor size reached $100 \mathrm{~mm}^{3}$, the experimental groups were intraperitoneally injected with cisplatin twice a week at a dosage of $15 \mathrm{mg} / \mathrm{kg}$. The control group was injected with the same volume of normal saline. After 4 weeks of cisplatin treatment, the tumors were removed and their volumes were calculated, then fixed with $4 \%$ paraformaldehyde for the following experiments.

Immunohistochemistry. Tumor tissues were fixed in $4 \%$ paraformaldehyde for $48 \mathrm{~h}$ at room temperature, embedded in paraffin for $48 \mathrm{~h}$ at room temperature and then $5-\mu \mathrm{m}$ thick sections were obtained. After deparaffinating, sections were stained with hematoxylin and eosin (H\&E) under standard $\mathrm{H} \& \mathrm{E}$ staining procedures. The expression of $\mathrm{Ki}-67$ and cleaved caspase- 3 was detected using monoclonal antibodies (anti-Ki-67 and anti-cleave-caspase-3; cat. nos. ab15580 and ab2302; 1:200; Abcam, Cambridge, MA, USA) and a horseradish peroxidase-conjugated anti-mouse or anti-rabbit secondary antibody (cat. nos. ab205719 and ab6721; 1:100; Abcam), followed by color development with diaminobenzidine tetrahydrochloride (DAB; Dako; Agilent Technologies, Inc., Santa Clara, CA, USA). The sections were counterstained with hematoxylin following DAB staining. The images were observed and analyzed under a microscope (Leica Microsystems GmbH, Wetzlar, Germany) with Image-Pro Plus version 6.0 (Media Cybernetics, Inc., Rockville, MD, USA). The magnification of H\&E sections was $\mathrm{x} 40$ and for immuohistochemical staining $\times 200$. The positive staining in images was quantified as integral optical density (IOD)/area, which was expressed as mean density. For each section, 3 fields were calculated and the average taken, and 6 sections were imaged in each group.

Statistical analysis. Results were expressed as the mean \pm standard deviation from three independent repeats, including the animal experiments. Statistical analyses were performed with Student's t-test or one-way analysis of variance followed by Duncan's post hoc test using SPSS 24.0 (IBM Corp., Armonk, NY, USA). $\mathrm{P}<0.05$ was considered to indicate a statistically significant difference.

\section{Results}

TGM2 is upregulated in cisplatin-resistant osteosarcoma cells. First, a cisplatin-resistant osteosarcoma cell line (termed here Saos2-CIS-R) was established based on the parental Saos2 osteosarcoma cells. To verify the chemoresistance of these cells, a cell viability assay was performed following treatment with different concentrations of cisplatin. The results demonstrated that Saos2-CIS-R cells had developed chemoresistance to cisplatin (Fig. 1). In the parental Saos2 cells, cell viability was gradually reduced with the increase of concentration of cisplatin and treating time (Fig. 1A and C-E). The cell viability of Saos2-CIS-R cells was significantly higher compared with Saos 2 cells, and the Saos2-CIS-R cells exhibited almost complete resistance to $1 \mu \mathrm{g} / \mathrm{ml}$ cisplatin (Fig. 1B-E). The apoptosis rate was detected using flow cytometry following treatment with $5 \mu \mathrm{g} / \mathrm{ml}$ cisplatin for $24 \mathrm{~h}$. The results indicated that Saos2-CIS-R cells had a lower apoptosis rate following cisplatin treatment compared with the parental Saos2 cells (Fig. 1F and G).

Next, TGM2 protein expression was measured by western blot analysis. As illustrated in Fig. 2A, TGM2 protein expression levels were significantly increased in Saos2-CIS-R cells compared with Saos 2 cells. The knockdown of TGM2 by shRNA (sh-TGM2) in Saos2-CIS-R cells and the overexpression of TGM2 (TGM2) in the parental Saos2 cells were also confirmed by western blot analysis (Fig. 2B).

TGM2 regulates the chemosensitivity of osteosarcoma cells to cisplatin. The abnormal expression of TGM2 in the Saos2-CIS-R and Saos 2 cell lines was hypothesized to be associated with the chemosensitivity. To examine the role of TGM2 in cisplatin sensitivity, a lentivirus-based gene knockdown and overexpression system was used in vitro. Cells were cultured for 7 days and the cell viability was measured over time in order to detect cell proliferation rates. In Saos2 cells, overexpression of TGM2 significantly promoted cell proliferation at all time points tested compared with control cells (Fig. 3A). In Saos2-CIS-R cells, knockdown of TGM2 significantly reduced cell proliferation from day 1 to 7 compared with scramble control (Fig. 3B). Following treatment with different concentrations of cisplatin for $24,48,72$ and $96 \mathrm{~h}$, cell viability results indicated that overexpression of TGM2 increased the chemoresistance of Saos 2 cells to cisplatin compared with control Saos2 cells (Fig. 3C). Similarly, knockdown of TGM2 in Saos2-CIS-R cells enhanced the chemosensitivity to cisplatin from 24 to $96 \mathrm{~h}$ compared with scramble control (Fig. 3D). Flow cytometry analysis further confirmed that overexpression of TGM2 in Saos2 cells reduced cisplatin-induced cell apoptosis, while knockdown of TGM2 in Saos2-CIS-R cells significantly increased cisplatin-induced cell apoptosis compared with scramble control (Fig. 4).

TGM2 regulates the MAPK pathway and caspase-3 activity in osteosarcoma cells. To further investigate the potential mechanism of TGM2 in regulating osteosarcoma cell chemosensitivity, the gene expression levels of Bax and BCL2 apoptosis regulator (Bcl-2) were measured in Saos2 and Saos2-CIS-R cells. Following treatment with $5 \mu \mathrm{g} / \mathrm{ml}$ cisplatin for $24 \mathrm{~h}, \mathrm{Bax}$ gene expression was downregulated, while Bcl-2 
A

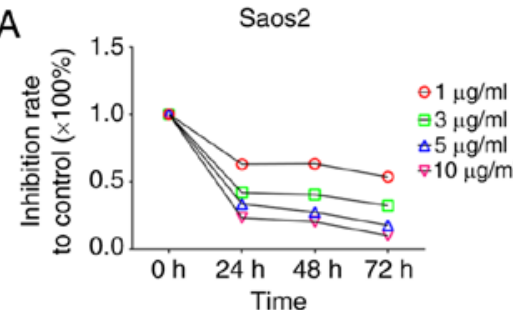

B

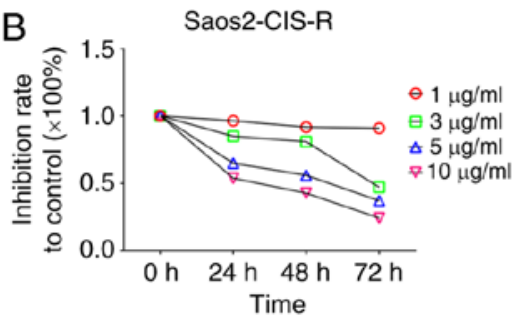

C

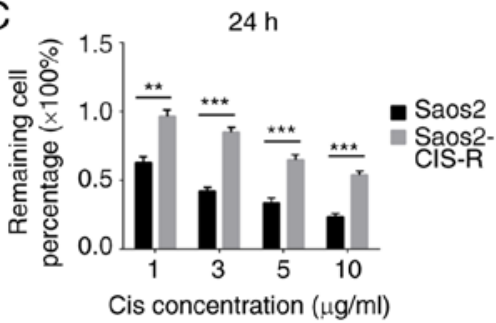

$\mathrm{F}$
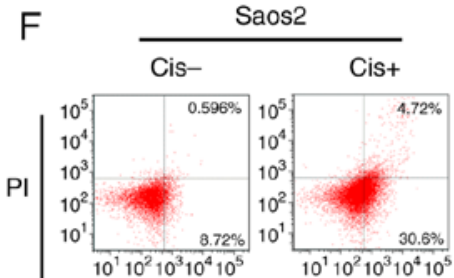

D

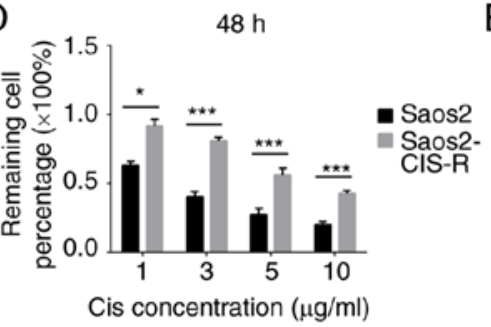

Cis concentration $(\mu \mathrm{g} / \mathrm{ml})$
E

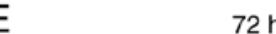

Annexin V-FITC

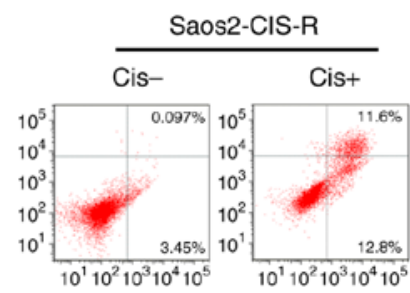

$10^{1} 10^{2} 10^{3} 10^{4} 10^{5}$
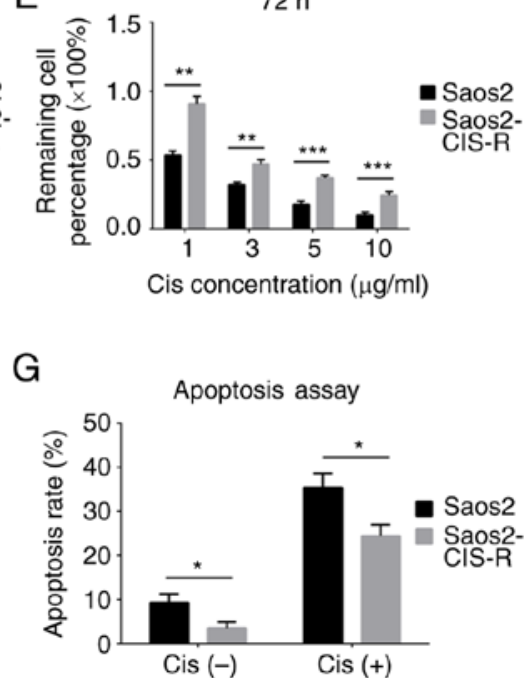

Figure 1. Establishment and identification of cisplatin-resistant cells. Saos2 cells were cultured and incubated with stepwisely increasing concentrations of cisplatin $(0.1-1 \mu \mathrm{g} / \mathrm{ml})$ for 1 year. Then cells growing in $1 \mu \mathrm{g} / \mathrm{ml}$ cisplatin were designated as cisplatin-resistant cells (Saos2-CIS-R). (A-E) Saos2-CIS-R and parental Saos 2 cells were treated with different concentrations of cisplatin for 24,48 and 72 h, and cell viability was measured by Cell Counting Kit- 8 assay. (F and G) Cell apoptosis was examined by Annexin V/PI double staining and analyzed by flow cytometry. Representative plots and quantification is shown. Data are presented as mean \pm standard deviation from at least three independent experiments. ${ }^{*} \mathrm{P}<0.05,{ }^{* *} \mathrm{P}<0.005$ and ${ }^{* * * *} \mathrm{P}<0.0005$, with comparisons indicated by lines. PI, propidium iodide; Cis, cisplatin; FITC, fluorescein isothiocyanate.

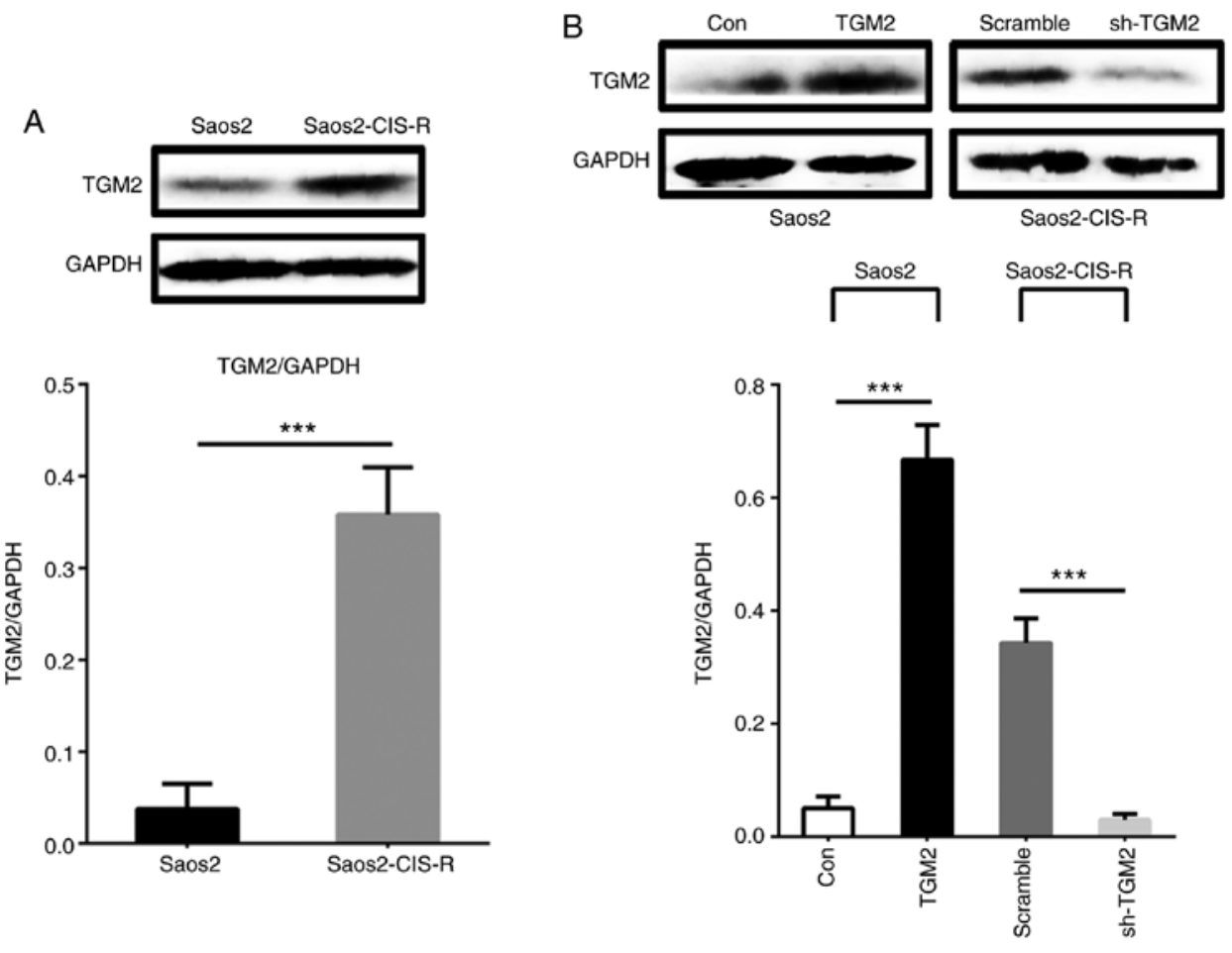

Figure 2. TGM2 expression in Saos2 and Saos2-CIS-R cells. (A) Protein expression levels of TGM2 in the parental Saos2 cells and in the cisplatin-resistant Saos2-CIS-R cells. (B) Saos2 cells were left untreated (control) or transduced with lentiviral particles overexpressing TGM2 (TGM2). Saos2-CIS-R cells were transduced with lentiviral particles containing shRNA against TGM2 (sh-TGM2) or a scramble control shRNA (scramble). TGM2 protein expression levels in the different cells were analyzed with western blotting. GAPDH was used as an internal control. Data are presented as the mean \pm standard deviation from at least three independent experiments. ${ }^{* * *} \mathrm{P}<0.0005$, with comparisons indicated by lines. TGM2, transglutaminase 2; sh, short hairpin; Con, control. 
A
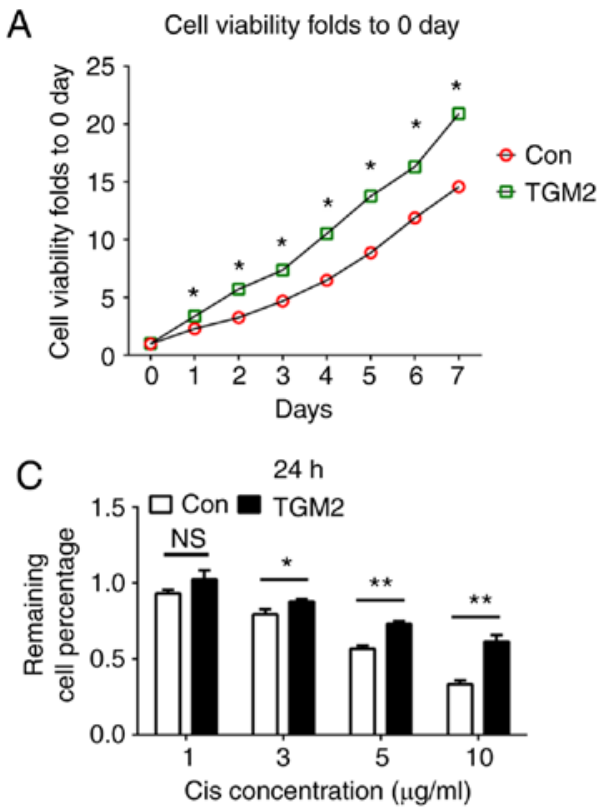

$48 \mathrm{~h}$

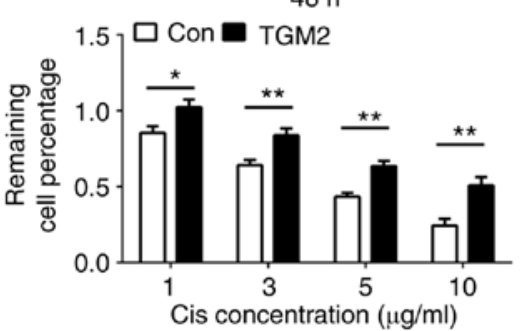

$72 \mathrm{~h}$

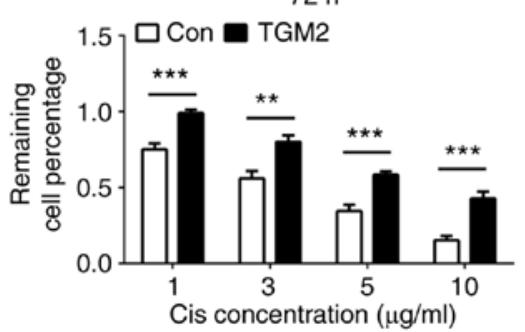

$96 \mathrm{~h}$

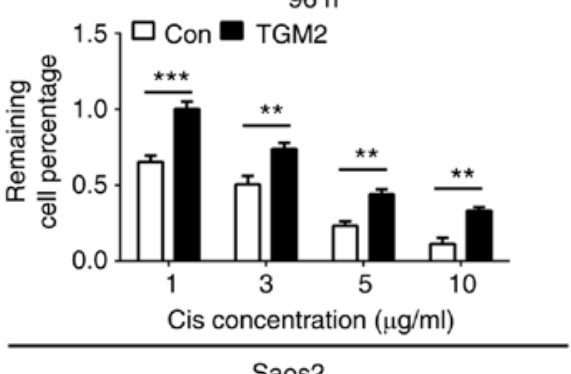

B

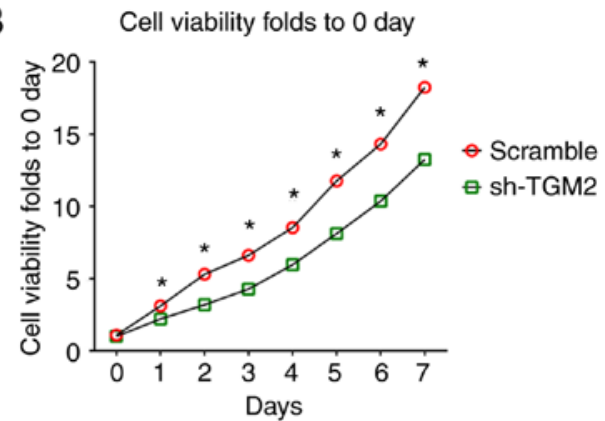

D

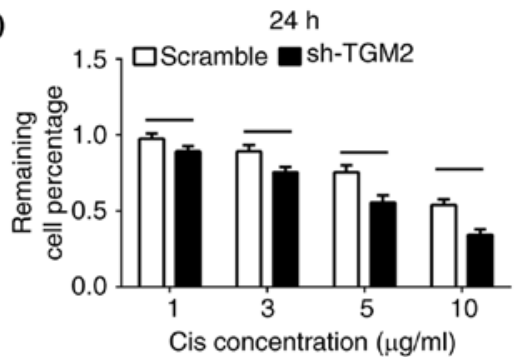

$48 \mathrm{~h}$

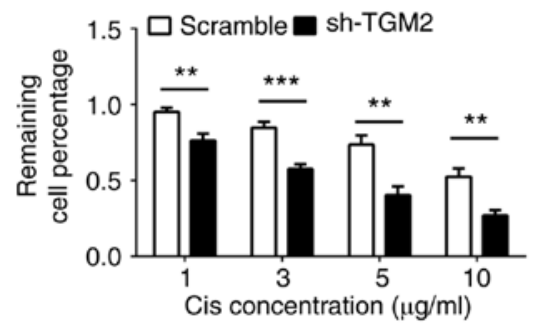

$72 \mathrm{~h}$

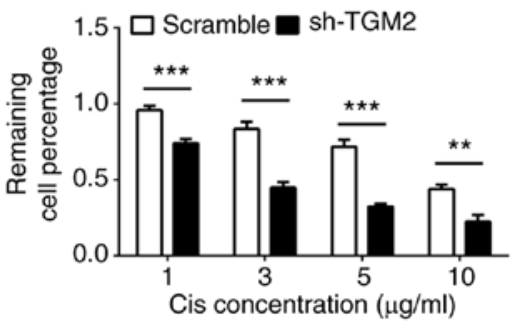

$96 \mathrm{~h}$

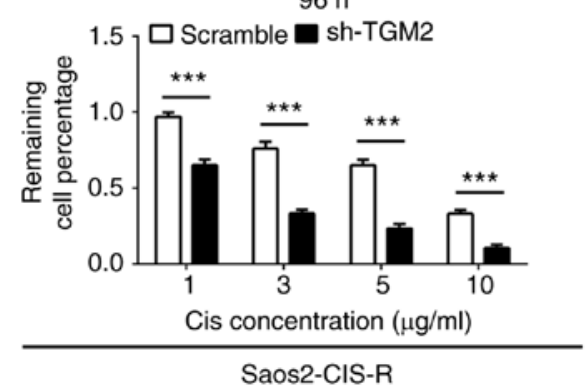

Figure 3. Cell proliferation and chemosensitivity following modulation of TGM2 expression in Saos2 and Saos2-CIS-R cells. (A) Saos2 and (B) Saos2-CIS-R cells were cultured for 7 days and the cell viability was measured by Cell Counting Kit- 8 assay. ${ }^{*} \mathrm{P}<0.05$ compared with control. (C) Saos 2 and (D) Saos $2-\mathrm{CIS}-\mathrm{R}$ cells were treated with different concentrations of cisplatin, and cell viability was detected at 24, 48, 72 and $96 \mathrm{~h}$. Data are presented as the mean \pm standard deviation from at least three independent experiments. ${ }^{*} \mathrm{P}<0.05,{ }^{* *} \mathrm{P}<0.005$ and ${ }^{* * *} \mathrm{P}<0.0005$, with comparisons indicated by lines. TGM2, transglutaminase 2 ; Con, control; sh, short hairpin; Cis, cisplatin.

gene expression was upregulated, in the TGM2-overexpressing Saos 2 cells compared with control Saos 2 cells (Fig. 5A). On the contrary, in sh-TGM2 cells, Bax gene expression was upregulated and Bcl-2 was downregulated compared with scramble control (Fig. 5B). Western blot analysis confirmed these results on the protein level; Bax protein expression was reduced in TGM2-overexpressing Saos 2 cells and increased in TGM2-knockdown Saos2-CIS-R cells; Bcl-2 expression exhibited the opposite results (Fig. 6A). The Akt and the MAPK signaling pathways and the caspase family of proteins are also 

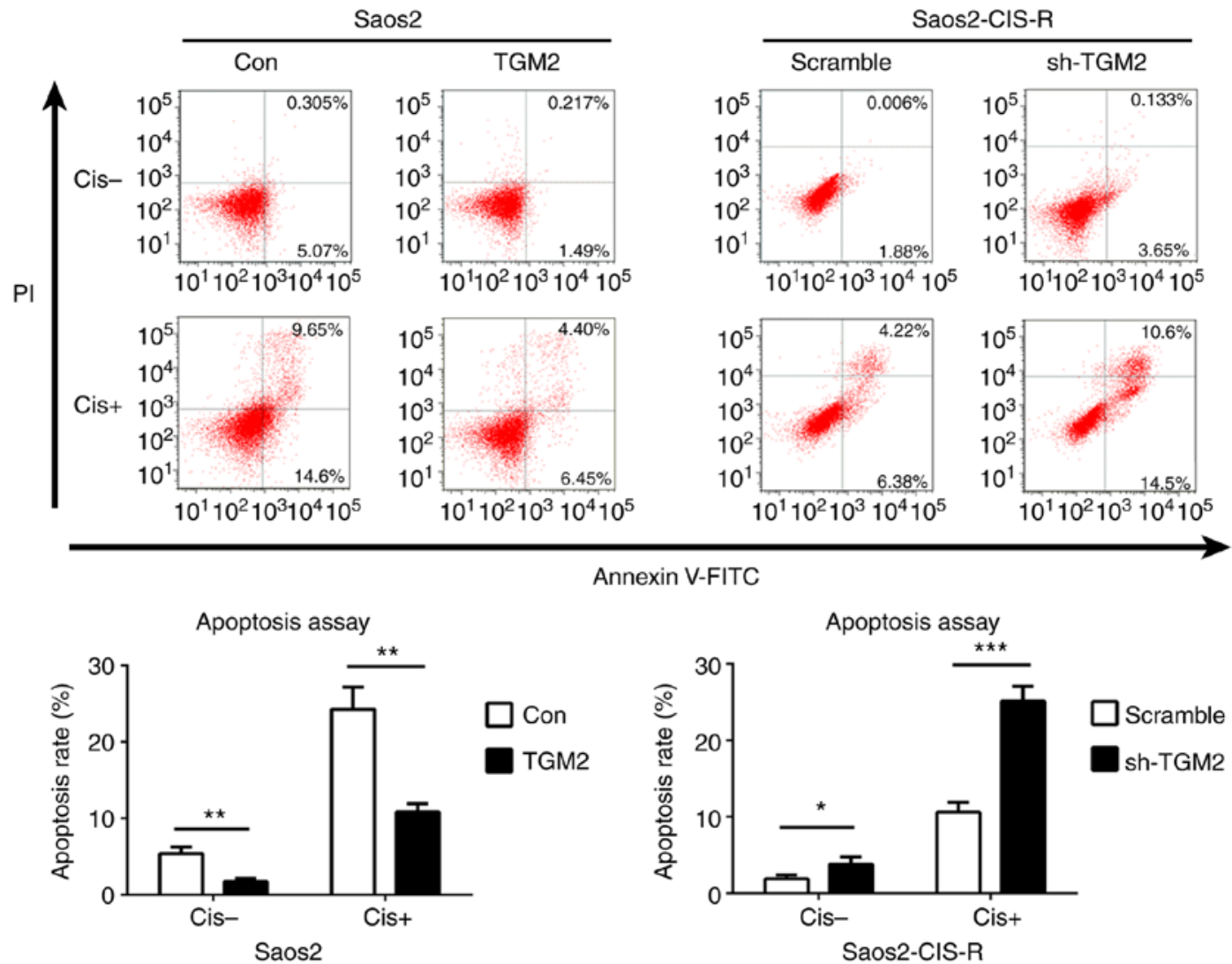

Annexin V-FITC

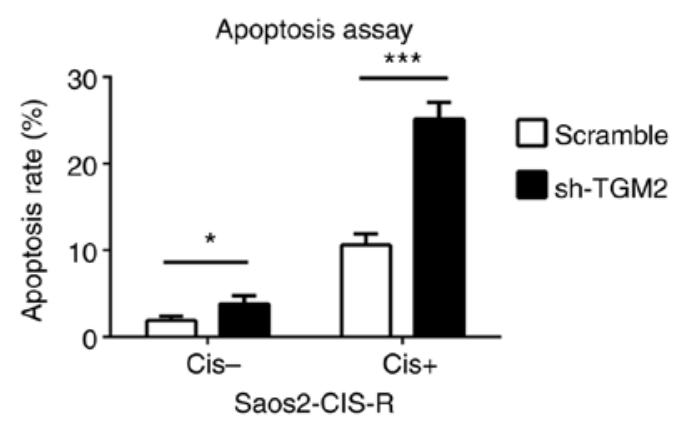

Figure 4. Cell apoptosis rates in Saos2 and Saos2-CIS-R cells. Following treatment with $5 \mu \mathrm{g} / \mathrm{ml}$ cisplatin for $24 \mathrm{~h}$, cell apoptosis was measured in Saos2 and Saos2-CIS-R cells by flow cytometry. Representative plots and quantification are shown. Data are presented as the mean \pm standard deviation from at least three independent experiments. ${ }^{*} \mathrm{P}<0.05,{ }^{* *} \mathrm{P}<0.005$ and ${ }^{* * *} \mathrm{P}<0.0005$, with comparisons indicated by lines. PI, propidium iodide; Cis, cisplatin; APC, allophycocyanin; TGM2, transglutaminase 2; Con, control; sh, short hairpin.
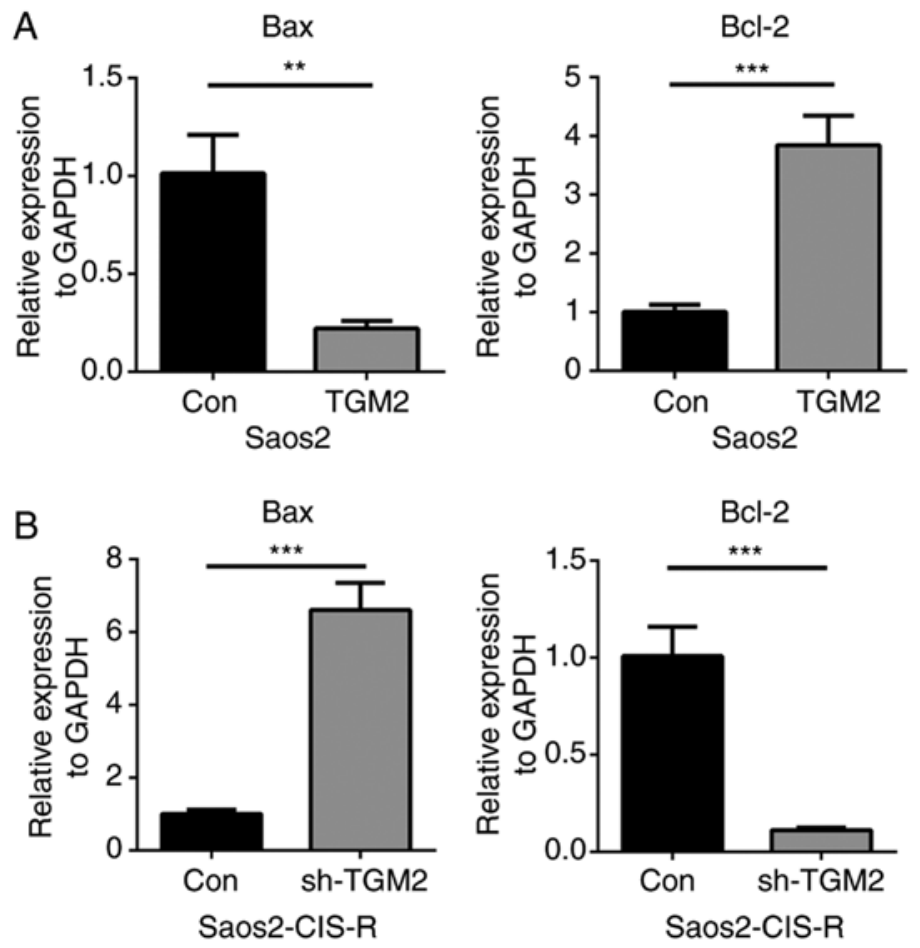

Figure 5. Bax and Bcl-2 mRNA expression in Saos2 and Sao2-CIS-R cells following modulation of TGM2 expression. Following treatment with $5 \mu \mathrm{g} / \mathrm{ml}$ cisplatin for $24 \mathrm{~h}$, the mRNA expression levels of Bax and Bcl-2 were detected by reverse transcription-quantitative polymerase chain reaction in (A) control or TGM2-overexpressing Saos2 cells, and (B) scramble or TGM2-knockdown Sao2-CIS-R cells. Gene expression was normalized to GAPDH. Data are presented as the mean \pm standard deviation from at least three independent experiments. ${ }^{* *} \mathrm{P}<0.005$ and ${ }^{* * * *} \mathrm{P}<0.0005$, with comparisons indicated by lines. Bax, BCL2 associated X; Bcl-2, BCL2 apoptosis regulator; TGM2, transglutaminase 2; Con, control; sh, short hairpin. 

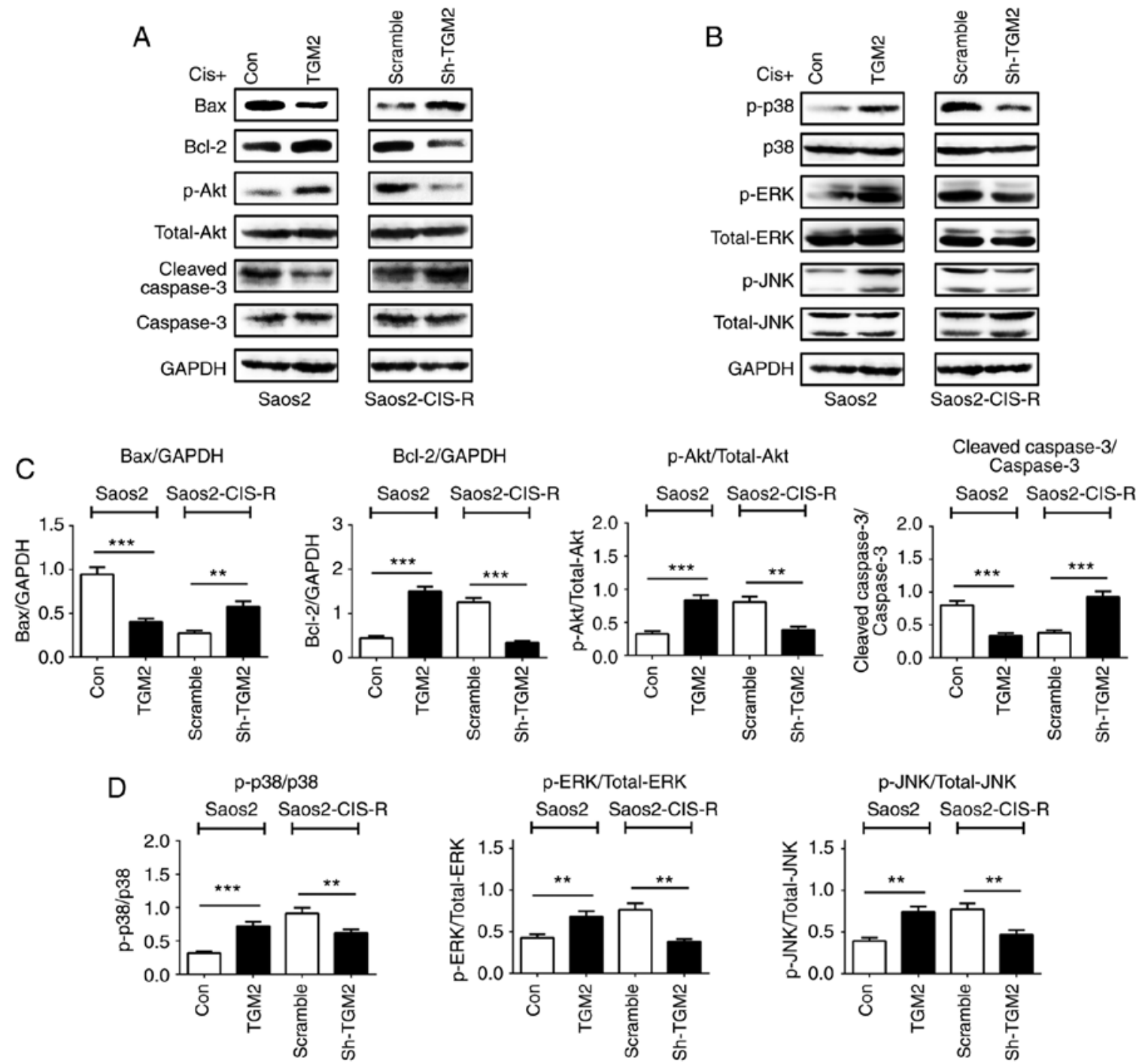

Figure 6. Expression of pathway-related proteins and caspase-3 in osteosarcoma cells. (A and B) Following treatment with $5 \mu \mathrm{g} / \mathrm{ml}$ cisplatin for $24 \mathrm{~h}$, protein expression levels of Bax, Bcl-2, Akt, p-Akt, cleaved caspase-3, caspase-3, p-p38, p38, p-ERK, ERK, p-JNK, JNK and GAPDH were all measured by western blotting. (C and D) Densitometry analysis of western blot results. Data are presented as the mean \pm standard deviation from at least three independent experiments. ${ }^{* *} \mathrm{P}<0.005$ and ${ }^{* * *} \mathrm{P}<0.0005$, with comparisons indicated by lines. Bax, BCL2 associated X; Bcl-2, BCL2 apoptosis regulator; Akt, AKT serine/threonine kinase; p-, phosphorylated; ERK, extracellular signal-regulated kinase; JNK, c-Jun N-terminal kinase; Cis, cisplatin; TGM2, transglutaminase 2; Con, control; sh, short hairpin.

known to have important roles in cell apoptosis. Therefore, the protein expression levels of Akt/phosphorylated (p-) Akt, caspase-3/cleaved caspase-3, p38/p-p38, extracellular signal-regulated kinase (ERK)/p-ERK and c-Jun N-terminal kinase (JNK)/p-JNK were analyzed by western blotting. The results indicated that, overexpression of TGM2 in Saos2 cells activated the Akt and MAPK pathways but inhibited the activity of caspase-3 (Fig. 6). By contrast, knockdown of TGM2 in the Saos2-CIS-R cells promoted the expression of cleaved caspase-3 and inhibited the activation of Akt and MAPK pathways (Fig. 6).

TGM2 knockdown enhances the chemosensitivity of osteosarcoma to cisplatin in vivo. Given that the in vitro experiments revealed encouraging results, the effect of TGM2 on chemosensitivity to cisplatin was further investigated in a mouse model of osteosarcoma. At the end of in vivo treatments, tumors were removed and their volumes were calculated. Tumor volumes were decreased in all groups following treatment with cisplatin. In the sh-TGM2 group, cisplatin treatment almost completely eliminated the tumors, and the femur remained intact (Fig. 7A). In the scramble control group, the tumors were still obvious, and the proximal femur was deformed, which suggests that these cells were chemoresistant. Quantification of the tumor volumes revealed that TGM2 knockdown resulted in significantly reduced tumor volumes compared with the scramble control group (Fig. 7B).

H\&E staining and immunohistochemistry analysis also indicated that knockdown of TGM2 enhanced the chemosensitivity to cisplatin. The H\&E staining revealed that the tumor mass was significantly decreased in the femurs of the sh-TGM2 group compared with scramble group (Fig. 7C). In addition, the scramble group displayed an increase of positive Ki-67 staining compared with the sh-TGM2 group without cisplatin treatment. However, following cisplatin treatment, Ki-67 staining was markedly reduced, and there was almost 
A
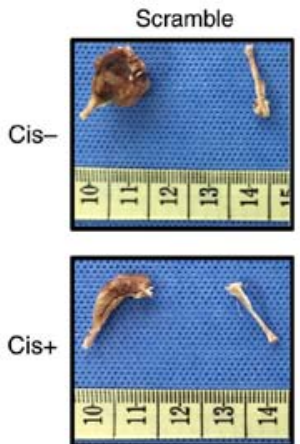

C

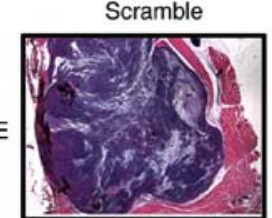

Ki-67

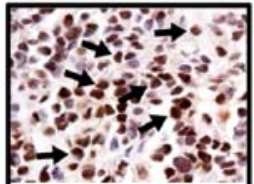

Sh-TGM2
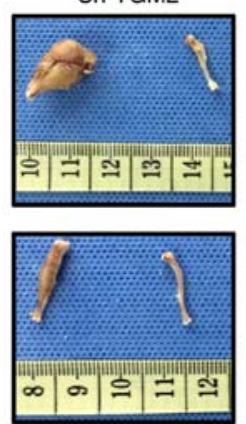

Sh-TGM2
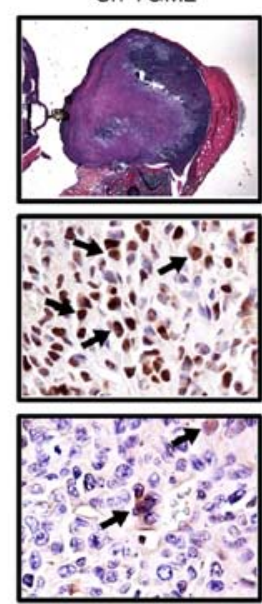

B
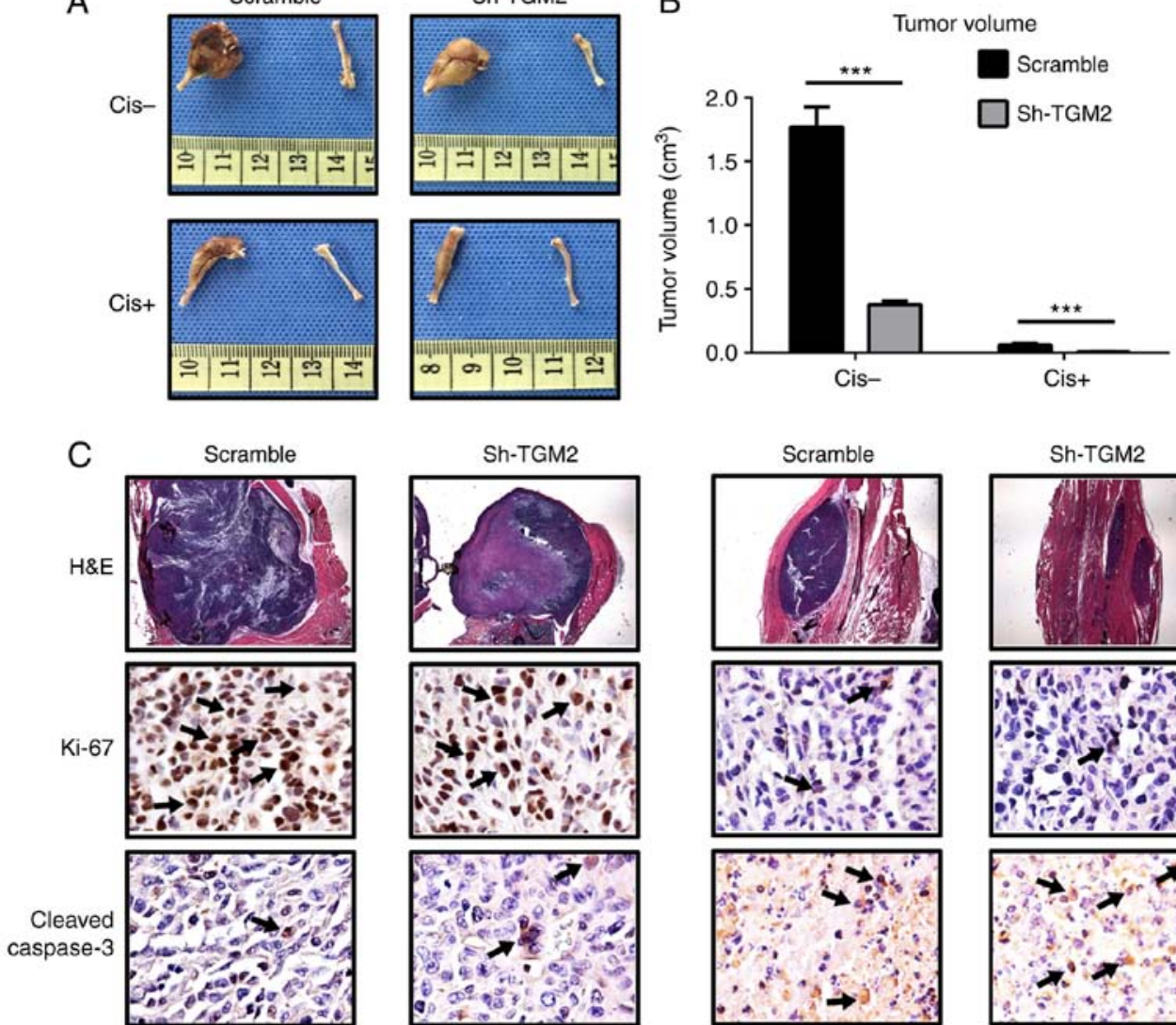

Cis-
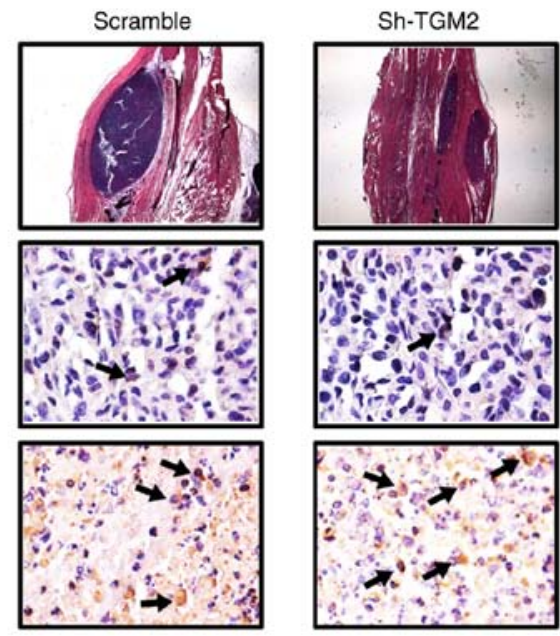

Cis+
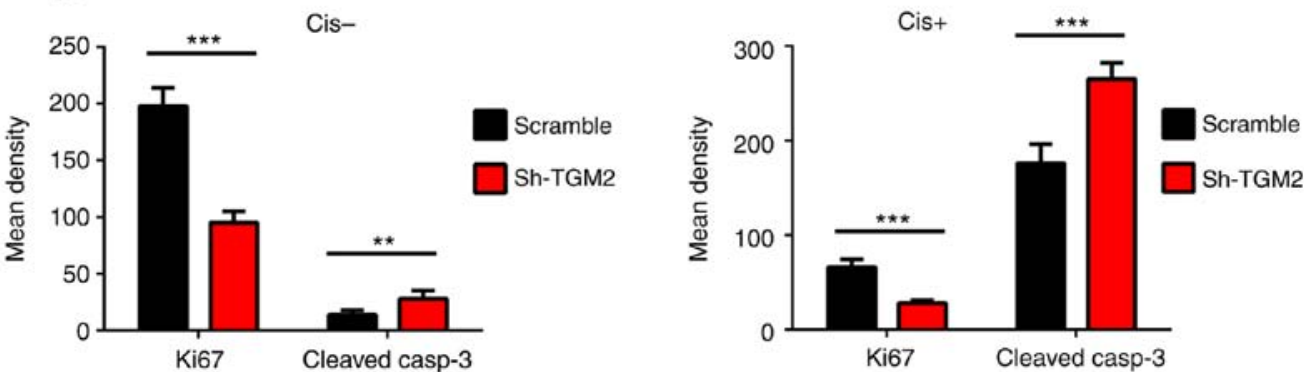

Figure 7. Cisplatin sensitivity of Saos2-CIS-R cells following TGM2 knockdown in vivo. Saos2-CIS-R cells (scramble and sh-TGM2) were injected into the proximal tibia of mice. When the tumor size reached $100 \mathrm{~mm}^{3}$, the mice were intraperitoneally injected with cisplatin (Cis+) or normal saline (Cis-). After 4 weeks of cisplatin treatment, the tumors were removed. (A) Representative tumor specimens from mice. (B) Tumor volumes were measured and calculated per group. (C) Tumor tissues were fixed and sections were stained for H\&E, and immunostained for Ki-67 and cleaved caspase-3. Representative images are shown for each experimental group. The magnification of H\&E section was $\mathrm{x} 40$ and for the immuohistochemical staining $\mathrm{x} 200$. Black arrows point to positive staining. (D) The mean density of Ki-67 and cleaved caspase-3 staining in tumor tissue sections was quantified. Data are presented as the mean \pm standard deviation from at least three independent experiments. ${ }^{* *} \mathrm{P}<0.005$ and ${ }^{* * *} \mathrm{P}<0.0005$, with comparisons indicated by lines. TGM2, transglutaminase 2 ; sh, short hairpin; Cis, cisplatin; H\&E, hematoxylin and eosin.

no positive staining in the sh-TGM2 group, compared with little staining in the scramble group (Fig. 7C; Ki-67 staining represented by arrows). Quantitative analysis of the results demonstrated that the sh-TGM2 group had significantly reduced $\mathrm{Ki}-67$ staining with or without cisplatin treatment compared with the scramble control (Fig. 7D). Cleaved caspase-3 staining was barely obvious before cisplatin treatment (Fig. 7C). In the sh-TGM2 group, a small amount of positive staining was observed (Fig. 7C; represented by arrows), while almost no positive staining was observed in the scramble group. Following cisplatin treatment, cleaved caspase-3 staining was remarkably increased, and there was more positive staining in the sh-TGM2 group (Fig. 7C). Quantitative analysis of the immunohistochemistry results confirmed that the sh-TGM2 group had significantly increased cleaved caspase-3 staining compared with the scramble group (Fig. 7D). These results demonstrated that knockdown of TGM2 in the Saos2-CIS-R cells suppressed the osteosarcoma cell proliferation and promoted cell apoptosis, thus enhancing the chemosensitivity to cisplatin.

\section{Discussion}

Chemotherapy is one of the principal modes of treatment for cancer, and cisplatin is a standard chemotherapy agent for osteosarcoma. However, chemoresistance of cisplatin has become a 
growing problem (17). Several mechanisms have been reported to be involved in drug chemoresistance, including mutation of drug targets and alterations in drug metabolism, while the detailed mechanisms have yet to be fully elucidated (18). Considering the complicated mechanisms of chemoresistance, the establishment of resistant cancer cell lines is crucial for studying the underlying mechanisms. In the present study, a cisplatin-resistant osteosarcoma cell line was established by exposure of osteosarcoma cells to a stepwisely increasing concentration of cisplatin (19).

The deregulation of apoptosis is one of the most important downstream mechanisms of chemoresistance in cancer. Changes in expression of apoptosis-related proteins, such as the Bcl-2 family and the caspase family of proteins, are associated with various cancers and responsible for resistance to chemotherapy (20). According to Zhao et al (21), Bcl-2 was markedly downregulated in a dose-dependent manner in cisplatin-treated Saos2 cells. Another study reported that gankyrin (P28GANK) conferred multidrug resistance by modulating expression of multidrug resistance protein 1 (MDR1), Bcl-2, and Bax in osteosarcoma cells (22). These studies indicated that regulation of Bax and Bcl-2 expression was an important mechanism of developing chemoresistance in osteosarcoma. Caspase- 3 is a key protein in the regulation of cell apoptosis and is involved in the formation of chemoresistance (23). TGM2 is also reported to have crucial roles in many processes including cell apoptosis (24). In multiple cancer cell lines, expression of TGM2 has been demonstrated to be associated with cisplatin and doxorubicin chemoresistance, and knockdown of TGM2 could increase chemosensitivity (25-27). A previous study indicated that TGM2 regulated cell apoptosis in a complicated manner: It not only promoted apoptosis but could also inhibit apoptosis (27). Recently, Wang et al (13) studied the role of TGM2 in the U2OS cell line. As their results demonstrated, TGM2 inhibited tumor cell apoptosis through downregulation of Bax and prevention of cytochrome $\mathrm{C}$ release (13). These results are also consistent with the experiments in the present study (Fig. 5). According to another study, increased expression of TGM2 was associated with epithelial-mesenchymal transition (EMT) in breast cancer and downregulation of TGM2 could reverse EMT and increase the chemosensitivity of breast cancer to docetaxel (28). In the present study, the gene expression of TGM2 was upregulated in Saos2-CIS-R cells, suggesting that gene regulation of TGM2 might be one of the mechanisms inducing chemoresistance. Furthermore, knockdown of TGM2 reversed the chemosensitivity of Saos2-CIS-R cells to cisplatin, and induced upregulation of Bax and caspase-3, and downregulation of Bcl-2. These results were consistent with previous reports $(13,25,26)$, indicating that TGM2 enhances chemoresistance via regulation of Bax, Bcl-2 and caspase-3.

Activation of the Akt and MAPK pathways has been observed in many malignancies and is considered to contribute to tumor cell survival $(29,30)$. A previous study has demonstrated that inhibition of the Akt pathway leads to the restoration of chemosensitivity in osteosarcoma cells (31). In addition, in vitro and in vivo studies have demonstrated that the Akt pathway has an important role in the adaptive resistance of tumors to chemotherapies, which is termed as 'oncogenic bypass' $(32,33)$. The MAPK pathway has also been demonstrated to have a key role in tumor cell survival and migration (34). Several drugs targeting the MAPK pathway have been reported to inhibit cell proliferation in osteosarcoma (35). The present study indicated that the Akt and MAPK pathways were activated in the drug-resistant cell line, and their activation was significantly inhibited following knockdown of TGM2. These results revealed that knockdown of TGM2 enhanced the chemosensitivity of osteosarcoma to cisplatin by inhibiting the activation of Akt and MAPK signaling.

In conclusion, our in vivo and in vitro experiments revealed that TGM2 was associated with the chemoresistance to cisplatin in osteosarcoma, and that knockdown of TGM2 enhanced osteosarcoma cell chemosensitivity. TGM2 might affect the chemosensitivity of osteosarcoma via regulation of the activation of MAPK and Akt pathways. Expression of Bcl-2, Bax and caspase-3 was also involved in the developing chemoresistance in osteosarcoma. The present findings suggest a potentially important role of TGM 2 in the regulation of osteosarcoma chemosensitivity. TGM2 may therefore serve as a potential therapeutic target for osteosarcoma.

\section{Acknowledgements}

Not applicable.

\section{Funding}

No funding was received

\section{Availability of data and materials}

The analyzed datasets generated during the study are available from the corresponding author on reasonable request.

\section{Authors' contributions}

CY conceived and designed the experiments, performed the experiments, analyzed the data, contributed reagents/materials/analytical tools, wrote the paper, prepared figures and tables, and reviewed drafts of the paper. JC performed the experiments, analyzed the data, contributed reagents/materials/analysis tools, and prepared figures and tables. FG performed the experiments. GW conceived and designed the experiments and reviewed drafts of the paper.

\section{Ethics approval and consent to participate}

All animal operations were approved by the Animal Ethics Committee of Linyi People's Hospital (Linyi, China; Approval no. 2016-A036).

\section{Patient consent for publication}

Not applicable.

\section{Competing interests}

The authors declare that they have no competing interests 


\section{References}

1. Mirabello L, Troisi RJ and Savage SA: Osteosarcoma incidence and survival rates from 1973 to 2004: Data from the surveillance, epidemiology, and end results program. Cancer 115: 1531-1543, 2009.

2. Bian ZY, Fan QM, Li G, Xu WT and Tang TT: Human mesenchymal stem cells promote growth of osteosarcoma: Involvement of interleukin- 6 in the interaction between human mesenchymal stem cells and Saos-2. Cancer Sci 101: 2554-2560, 2010.

3. Tu B, Du L, Fan QM, Tang Z and Tang TT: STAT3 activation by IL- 6 from mesenchymal stem cells promotes the proliferation and metastasis of osteosarcoma. Cancer Lett 325: 80-88, 2012.

4. Dieudonné FX, Marion A, Haÿ E, Marie PJ and Modrowski D: High Wnt signaling represses the proapoptotic proteoglycan syndecan-2 in osteosarcoma cells. Cancer Res 70: 5399-5408, 2010.

5. Dasari S and Tchounwou PB: Cisplatin in cancer therapy: Molecular mechanisms of action. Eur J Pharmacol 740: 364-378, 2014.

6. He H, Ni J and Huang J: Molecular mechanisms of chemoresistance in osteosarcoma (Review). Oncol Lett 7: 1352-1362, 2014.

7. Longley DB and Johnston PG: Molecular mechanisms of drug resistance. J Pathol 205: 275-292, 2005.

8. Hsieh YF, Liu GY, Lee YJ, Yang JJ, Sándor K, Sarang Z, Bononi A, Pinton P, Tretter L, Szondy Z and Tsay GJ: Transglutaminase 2 contributes to apoptosis induction in Jurkat T cells by modulating $\mathrm{Ca}^{2+}$ homeostasis via cross-linking RAP1GDS1. PLoS One 8: e81516, 2013

9. Lauzier A, Charbonneau M, Paquette M, Harper K and Dubois CM: Transglutaminase 2 cross-linking activity is linked to invadopodia formation and cartilage breakdown in arthritis Arthritis Res Ther 14: R159, 2012.

10. Singer CF, Hudelist G, Walter I, Rueckliniger E, Czerwenka K, Kubista $\mathrm{E}$ and Huber AV: Tissue array-based expression of transglutaminase-2 in human breast and ovarian cancer. Clin Exp Metastasis 23: 33-39, 2006.

11. Park KS, Kim HK, Lee JH, Choi YB, Park SY, Yang SH, Kim SY and Hong KM: Transglutaminase 2 as a cisplatin resistance marker in non-small cell lung cancer. J Cancer Res Clin Oncol 136: 493-502, 2010.

12. Hwang JY, Mangala LS, Fok JY, Lin YG, Merritt WM, Spannuth WA, Nick AM, Fiterman DJ, Vivas-Mejia PE, Deavers MT, et al: Clinical and biological significance of tissue transglutaminase in ovarian carcinoma. Cancer Res 68 5849-5858, 2008.

13. Wang W, Li X, Han XZ, Meng FB, Wang ZX, Zhai YQ and Zhou DS: Transglutaminase-2 is involved in cell apoptosis of osteosarcoma cell line U2OS under hypoxia condition. Cell Biochem Biophys 72: 283-288, 2015.

14. Mehta K: High levels of transglutaminase expression in doxorubicin-resistant human breast carcinoma cells. Int J Cancer 58 400-406, 1994

15. Antonyak MA, Jansen JM, Miller AM, Ly TK, Endo M and Cerione RA: Two isoforms of tissue transglutaminase mediate opposing cellular fates. Proc Natl Acad Sci USA 103 18609-18614, 2006.

16. Livak KJ and Schmittgen TD: Analysis of relative gene expression data using real-time quantitative PCR and the $2^{-\Delta \Delta C_{\mathrm{T}}}$ method. Methods 25: 402-408, 2001.

17. Li S, Sun W, Wang H, Zuo D, Hua Y and Cai Z: Research progress on the multidrug resistance mechanisms of osteosarcoma chemotherapy and reversal. Tumour Biol 36: $1329-1338,2015$

18. Holohan C, Van Schaeybroeck S, Longley DB and Johnston PG: Cancer drug resistance: An evolving paradigm. Nat Rev Cancer 13: 714-726, 2013.
19. Asada N, Tsuchiya H, Ueda Y and Tomita K: Establishment and characterization of an acquired cisplatin-resistant subline in a human osteosarcoma cell line. Anticancer Res 18: 1765-1768, 1998.

20. Letai AG: Diagnosing and exploiting cancer's addiction to blocks in apoptosis. Nat Rev Cancer 8: 121-132, 2008.

21. Zhao Z, Tao L, Shen C, Liu B, Yang Z and Tao H: Silencing of Barkor/ATG14 sensitizes osteosarcoma cells to cisplatin-induced apoptosis. Int J Mol Med 33: 271-276, 2014.

22. Wang G, Rong J, Zhou Z and Duo J: Novel gene P28GANK confers multidrug resistance by modulating the expression of MDR-1, Bcl-2 and Bax in osteosarcoma cells. Mol Biol 44: 1010-1017, 2010.

23. Botham RC, Roth HS, Book AP, Roady PJ, Fan TM and Hergenrother PJ: Small-molecule procaspase-3 activation sensitizes cancer to treatment with diverse chemotherapeutics. ACS Cent Sci 2: 545-559, 2016.

24. Lee HJ and Lee CH: Transglutaminase-2 is involved in expression of osteoprotegerin in MG-63 osteosarcoma cells. Biomol Ther 21: 204-209, 2013.

25. Cao L, Petrusca DN, Satpathy M, Nakshatri H, Petrache and Matei D: Tissue transglutaminase protects epithelial ovarian cancer cells from cisplatin-induced apoptosis by promoting cell survival signaling. Carcinogenesis 29: 1893-1900, 2008.

26. Han JA and Park SC: Reduction of transglutaminase 2 expression is associated with an induction of drug sensitivity in the PC-14 human lung cancer cell line. J Cancer Res Clin Oncol 125: 89-95, 1999.

27. Nurminskaya MV and Belkin AM: Cellular functions of tissue transglutaminase. Int Rev Cell Mol Biol 294: 1-97, 2012.

28. He W, Sun Z and Liu Z: Silencing of TGM2 reverses epithelial to mesenchymal transition and modulates the chemosensitivity of breast cancer to docetaxel. Exp Ther Med 10: 1413-1418, 2015.

29. Zöllinger A, Stühmer T, Chatterjee M, Gattenlöhner S, Haralambieva E, Müller-Hermelink HK, Andrulis M, Greiner A, Wesemeier C, Rath JC, et al: Combined functional and molecular analysis of tumor cell signaling defines 2 distinct myeloma subgroups: Akt-dependent and Akt-independent multiple myeloma. Blood 112: 3403-3411, 2008.

30. Peng DJ, Wang J, Zhou JY and Wu GS: Role of the Akt/mTOR survival pathway in cisplatin resistance in ovarian cancer cells. Biochem Biophys Res Commun 394: 600-605, 2010.

31. Wang $\mathrm{H}$, Luo QF, Peng AF, Long XH, Wang TF, Liu ZL, Zhang GM, Zhou RP, Gao S, Zhou Y, et al: Positive feedback regulation between Akt phosphorylation and fatty acid synthase expression in osteosarcoma. Int J Mol Med 33: 633-639, 2014

32. Wheeler DL, Huang S, Kruser TJ, Nechrebecki MM, Armstrong EA, Benavente S, Gondi V, Hsu KT and Harari PM: Mechanisms of acquired resistance to cetuximab: Role of HER (ErbB) family members. Oncogene 27: 3944-3956, 2008.

33. Sergina NV, Rausch M, Wang D, Blair J, Hann B, Shokat KM and Moasser MM: Escape from HER-family tyrosine kinase inhibitor therapy by the kinase-inactive HER3. Nature 445 : 437-441, 2007

34. Liao YX, Zhou CH, Zeng H, Zuo DQ, Wang ZY, Yin F, Hua YQ and Cai ZD: The role of the CXCL12-CXCR4/CXCR7 axis in the progression and metastasis of bone sarcomas (Review). Int J Mol Med 32: 1239-1246, 2013.

35. Cheng DD, Zhu B, Li SJ, Yuan T, Yang QC and Fan CY Down-regulation of RPS9 inhibits osteosarcoma cell growth through inactivation of MAPK signaling pathway. J Cancer 8: 2720-2728, 2017

(i) $($ - This work is licensed under a Creative Commons Attribution-NonCommercial-NoDerivatives 4.0 International (CC BY-NC-ND 4.0) License. 